\title{
Secondary Metabolites by Chemical Screening, 3 [1] Side-Chain Modification of Elaiophylin
}

\author{
Peter Hammann* and Gerhard Kretzschmar \\ Hoechst AG, Postfach 8003 20, D-6230 Frankfurt am Main 80 \\ Z. Naturforsch. 45b, 515-517 (1990); received September 29, 1989 \\ Elaiophylin, Macrodiolid, Secondary Metabolites, Chemical Screening \\ Base catalyzed deglycosidation of elaiophylin leads to a reactive enone system. Following \\ addition of thiols yield side-chain modified products of the title compound.
}

Elaiophylin (1) is a macrodiolid antibiotic which was first isolated from Streptomyces melanosporus [2]. First it was attempted to elucidate the structure by an alkaline degradation of 1 [3-7]. Under acidic conditions the cleavage of the carbohydrate units was achieved [6]. The modification of the side-chain by glycosidation or even acylation was not possible because of steric hindrance of $\mathrm{OH}-13$ [8].

During our work on structure modification of elaiophylin (1) we found reaction conditions for a controlled alkaline deglycosidation. Reaction of $\mathbf{1}$ with $\mathrm{KHCO}_{3}$ in water/ethanol/ethyl acetate leads to a $\beta$-elimination with $\mathrm{L}$-deoxyfucose as leaving group. The corresponding monoenone 2 was obtained in $34 \%$ and the dienone 3 in $52 \%$ yield. The trans-stereochemistry of the 12-double bond was confirmed by the coupling constant $J_{1213}=$ $15.9 \mathrm{~Hz}$ in the ${ }^{1} \mathrm{H}$ NMR spectrum. Obviously, the elimination is faster than the retro-aldol cleavage under these conditions. The trans-configuration of the double bond indicates that the hemiacetale is opened prior to elimination of L-deoxyfucose. The base attack at $\mathrm{C}-12$, instead of deprotonation at $\mathrm{OH}-9$, which would be the first step of the retroaldol cleavage, can be explained by steric hindrance through hydrogen bonding of $\mathrm{OH}-9$ to the carbonyl group at C-1'.

In the ${ }^{13} \mathrm{C}$ NMR spectra of $\mathbf{3}$ only 21 signals were observed because of the $\mathrm{C}_{2}$-symmetry. Compound $\mathbf{2}$ in contrast exhibited 48 signals because of its unsymmetry. Hereby 27 signals of 1 and 21 signals of $\mathbf{3}$ could be detected in the ${ }^{13} \mathrm{C}$ NMR spectra of 2.

The hydration of $\mathbf{2}$ and $\mathbf{3}$ with $\mathrm{Pd} / \mathrm{H}_{2}$ results in the formation of 13,13'-dideoxyoctahydroelaio-

\footnotetext{
* Reprint requests to Dr. P. Hammann.

Verlag der Zeitschrift für Naturforschung, D-7400 Tübingen 0932-0776/90/0400-0515/\$01.00/0
}

phylidene (5) and the corresponding unsymmetric product 4 in $92 \%$ and $85 \%$ yield, respectively.

Addition of thiols to the enone-system in $\mathbf{5}$ offers the possibility to synthesize modified sidechains in elaiophylidene for the first time. When $\mathbf{5}$ was refluxed with ethanethiol or mercaptoethanol in THF in the presence of triethylamine as catalyst, 6 and 7 were obtained in $82 \%$ and $74 \%$ yield. The $\beta$-stereochemistry of the thio-groups at C-13 was based on the coupling constants of $\mathrm{H}_{\mathrm{ax}}-13$ in the ${ }^{1} \mathrm{H}$ NMR spectra. For example with 6 the pseudo sextett of $\mathrm{H}-13$ could be explained by $J_{\mathrm{Hax}-12 \mathrm{Hax}-13}=12.5 \mathrm{~Hz}, J_{\mathrm{Hax}-14 \mathrm{Hax}-13}=12.5 \mathrm{~Hz}$ and $J_{\mathrm{Heq}-12 \mathrm{Hax}-13}=3.6 \mathrm{~Hz}$.

Table I. ${ }^{13} \mathrm{C}$ NMR data of elaiophylin derivatives (chemical shifts in ppm in pyridine-d $\mathrm{d}_{5}$ at $75.5 \mathrm{MHz}$ ).

\begin{tabular}{|c|c|c|c|c|}
\hline & 3 & 5 & 6 & 7 \\
\hline 1 & 168.0 & 174.7 & 168.5 & 168.5 \\
\hline 2 & 122.1 & 34.4 & 122.1 & 122.0 \\
\hline 3 & 145.3 & $24.8 *$ & 145.4 & 145.3 \\
\hline 4 & 131.4 & $24.5^{*}$ & 131.5 & 131.5 \\
\hline 5 & 145.0 & 32.8 & 145.0 & 145.0 \\
\hline 6 & 42.2 & 34.0 & 42.0 & 41.9 \\
\hline 7 & 76.8 & 77.1 & 77.0 & 76.9 \\
\hline 8 & 37.6 & 42.9 & 37.3 & 37.3 \\
\hline 9 & 72.2 & 70.5 & 70.8 & 70.8 \\
\hline 10 & 46.7 & 38.4 & 43.2 & 43.1 \\
\hline 11 & 202.7 & 98.3 & 99.2 & 99.1 \\
\hline 12 & 131.6 & 33.3 & 42.7 & 42.9 \\
\hline 13 & 148.3 & 24.6 & 41.2 & 41.6 \\
\hline 14 & 52.8 & 43.9 & 47.8 & 47.9 \\
\hline 15 & 68.9 & 70.0 & 68.3 & 68.3 \\
\hline 16 & 22.1 & 20.0 & 20.1 & 20.0 \\
\hline 17 & 15.7 & 16.1 & 15.7 & 15.6 \\
\hline 18 & 10.1 & 9.4 & 9.8 & 9.7 \\
\hline 19 & 9.1 & 7.3 & 7.5 & 7.4 \\
\hline 20 & 24.3 & $24.8 *$ & 21.3 & 21.3 \\
\hline 21 & 12.2 & 10.9 & 9.7 & 9.6 \\
\hline $1^{\prime}$ & & & 23.9 & 15.3 \\
\hline $2^{\prime}$ & & & 32.9 & 62.4 \\
\hline
\end{tabular}

* These signals may be interchanged. 


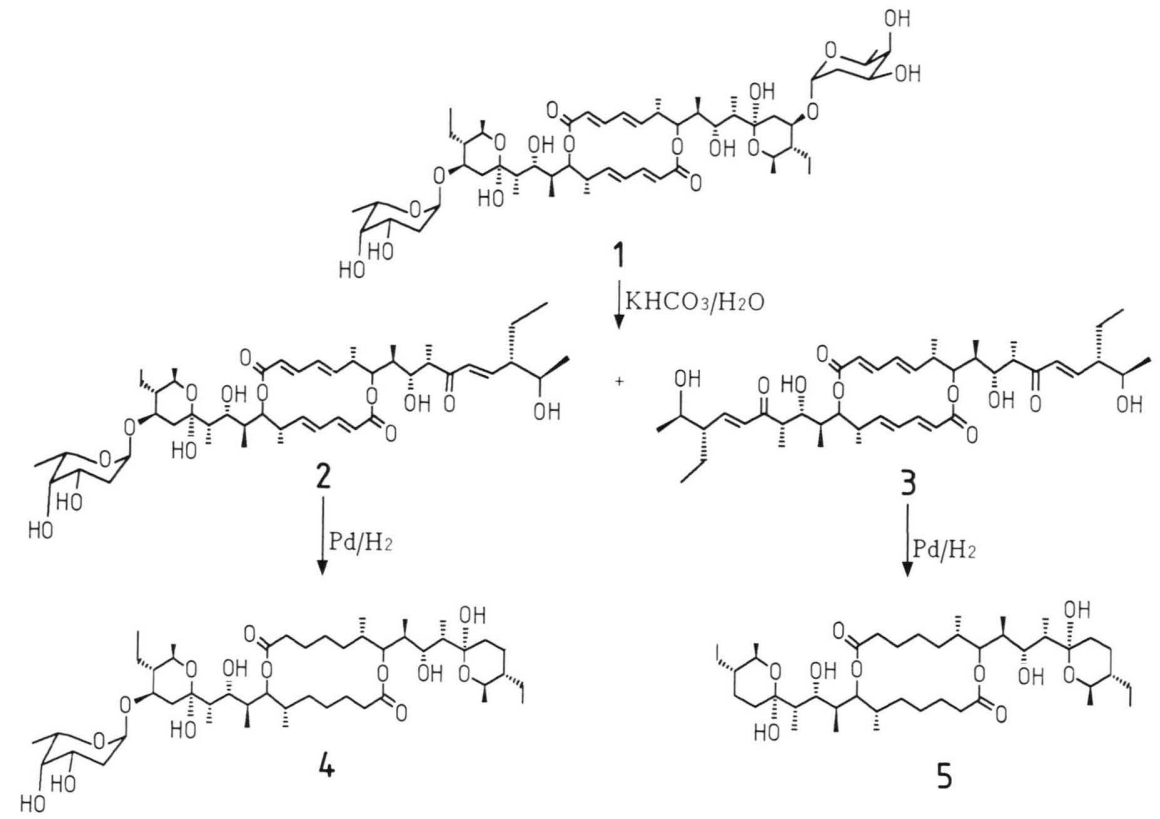

The alkaline deglycosidation of the macrolide elaiophylin $\mathbf{1}$ is a convenient method to produce valuaiole intermediates on preparative scale allowing further modification.

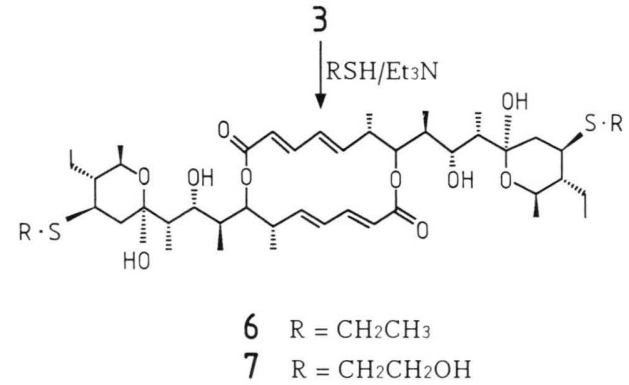

\section{Experimental}

Melting points were determined with a Reichert hot stage microscope and are not corrected. NMR spectra were recorded on a Bruker AM 300 with TMS as internal standard. FAB-mass spectra were taken by a Kratos MS 50 with 3-nitrobenzylalcohol as matrix. Optical rotation was measured with a Perkin Elmer spectrometer 241.

\section{Deglycosidation to $\mathbf{2}$ and $\mathbf{3}$}

$10 \mathrm{~g}(9.8 \mathrm{mmol}) \mathbf{1}$ was refluxed in a mixture containing $150 \mathrm{ml}$ of water, $50 \mathrm{ml}$ of ethanol and
$70 \mathrm{ml}$ of ethyl acetate with $25 \mathrm{~g}$ of potassium-hydrogencarbonate for $6 \mathrm{~h}$. The organic solvents were evaporated and the water phase was extracted four times with ethyl acetate. The organic phase was evaporated and the residue chromatographed on silica gel using chloroform/methanol $(40: 1)$ as eluent; yield $2.9 \mathrm{~g}(34 \%) 2$ and $3.7 \mathrm{~g}(52 \%) 3$.

2: $[a]_{20}^{\mathrm{D}}+13^{\circ}\left(\mathrm{c}=1, \mathrm{CH}_{3} \mathrm{OH}\right)$, positive FAB-MS $m / z=899\left(\mathrm{MNa}^{+}, 100 \%\right)$.

$\mathrm{C}_{48} \mathrm{H}_{76} \mathrm{O}_{14}(877.1)$
Calcd $\mathrm{C} 65.7$
Found $\mathrm{C} 65.0 \mathrm{H} 8.5$,

3: $[\alpha]_{20}{ }^{\mathrm{D}}+107^{\circ}\left(\mathrm{c}=1, \mathrm{CH}_{3} \mathrm{OH}\right)$, positive FABMS $m / z=751\left(\mathrm{MNa}^{+}, 100 \%\right),{ }^{1} \mathrm{H}$ NMR (pyridine- $\left.\mathrm{d}_{5}\right) \delta=0.8(\mathrm{t}, 3 \mathrm{H}, J=7.4 \mathrm{~Hz}, \mathrm{H}-21), 1.0(\mathrm{~d}$, $3 \mathrm{H}, J=6.6 \mathrm{~Hz}, \mathrm{H}-17), 1.15(\mathrm{~d}, 3 \mathrm{H}, J=6.9 \mathrm{~Hz}$, $\mathrm{H}-18), 1.23(\mathrm{~d}, 3 \mathrm{H}, J=6.2 \mathrm{~Hz}, \mathrm{H}-16), 1.4(\mathrm{~d}, 3 \mathrm{H}$, $J=6.9 \mathrm{~Hz}, \mathrm{H}-19), 1.6(\mathrm{~m}, 1 \mathrm{H}, \mathrm{H}-20), 1.75(\mathrm{~m}, 1 \mathrm{H}$, $\mathrm{H}-20), 2.1$ (m, 1 H, H-14), 2.3 (m, 1 H, H-8), 2.6 $(\mathrm{m}, 1 \mathrm{H}, \mathrm{H}-6), 3.35(\mathrm{~d}, 1 \mathrm{H}, J=2.9 \mathrm{~Hz}$ and $J=$ $6.9 \mathrm{~Hz}, \mathrm{H}-10), 4.07(\mathrm{~m}, 1 \mathrm{H}, \mathrm{H}-15), 4.32(\mathrm{~d}, \mathrm{~d}, 1 \mathrm{H}$, $J=8.3 \mathrm{~Hz}, J=2.0 \mathrm{~Hz}, \mathrm{H}-9), 5.65-5.85(\mathrm{~m}, 3 \mathrm{H}$, $\mathrm{H}-2, \mathrm{H}-5, \mathrm{H}-7), 6.15(\mathrm{~d}, \mathrm{~d}, 1 \mathrm{H}, J=11.3 \mathrm{~Hz}, J=$ $15.3 \mathrm{~Hz}, \mathrm{H}-4), 6.4(\mathrm{~d}, 1 \mathrm{H}, J=15.9 \mathrm{~Hz}, \mathrm{H}-12), 7.2$ (m, $2 \mathrm{H}, \mathrm{H}-3, \mathrm{H}-13$ ) ppm.

$$
\begin{aligned}
& \mathrm{C}_{42} \mathrm{H}_{64} \mathrm{O}_{10}(728.9) \\
& \text { Calcd } \mathrm{C} 69.2 \\
& \text { Found } \mathrm{C} 6.9 .9
\end{aligned}
$$




\section{Hydrogenation to $\mathbf{4}$ and $\mathbf{5}$}

$1 \mathrm{~g}$ of $\mathbf{2}$ or $\mathbf{3}$ was dissolved in $50 \mathrm{ml}$ of methanol and $400 \mathrm{mg}$ of palladium on charcoal (Merck) was added. The hydrogenation was carried out at room temperature for $2 \mathrm{~h}$. The reaction mixture was filtered and concentrated and the residue was chromatographed on silica gel with chloroform/ methanol (40:1). The yield of $\mathbf{4}$ was $92 \%$ and that of 5 was $85 \%$.

4: positive FAB-MS $m / z=909\left(\mathrm{MNa}^{+}\right)$.

$$
\mathrm{C}_{48} \mathrm{H}_{86} \mathrm{O}_{14}(887.2)
$$

Calcd C 65.0 H 9.8,

Found C 65.4 H 9.3.

5: $[\alpha]_{20}^{\mathrm{D}}+26^{\circ}\left(\mathrm{c}=1, \mathrm{CHCl}_{3}\right)$, m.p. $152-153{ }^{\circ} \mathrm{C}$, positive FAB-MS $m / z=763\left(\mathrm{MNa}^{+}\right),{ }^{1} \mathrm{H}$ NMR (pyridine- $\left.\mathrm{d}_{5}\right) \delta=4.0(\mathrm{~m}, 1 \mathrm{H}, \mathrm{H}-15), 4.5(\mathrm{~m}, 1 \mathrm{H}$, $\mathrm{H}-9), 5.6$ (d, $1 \mathrm{H}, J=2.5 \mathrm{~Hz}, \mathrm{H}-7$ ).

$$
\begin{gathered}
\mathrm{C}_{42} \mathrm{H}_{76} \mathrm{O}_{10}(741.1) \\
\text { Calcd } \mathrm{C} 68.1 \\
\text { Found } \mathrm{C} 67.6 \mathrm{H} 10.3,
\end{gathered}
$$

\section{Addition of thiols to 6 and 7}

$1 \mathrm{~g} 3$ was refluxed in a mixture containing $20 \mathrm{ml}$ of THF, $1 \mathrm{ml}$ of thiol and $0.5 \mathrm{ml}$ of triethylamine for $6 \mathrm{~h}$. The reaction mixture was concentrated and chromatographed on silica gel with chloro form/methanol $(40: 1)$. The yield of 6 was $82 \%$ and that of 7 was $74 \%$.

6: positive FAB-MS $m / z=874\left(\mathrm{MNa}^{+}, 100 \%\right)$, ${ }^{1} \mathrm{H}$ NMR (pyridine- $\left.\mathrm{d}_{5}\right) \delta=2.6(\mathrm{~m}, 1 \mathrm{H}, \mathrm{H}-6), 2.8$ (d, d, $J=12.8 \mathrm{~Hz}$ and $J=3.6 \mathrm{~Hz}, 1 \mathrm{H}, \mathrm{H}-12), 3.4$ (pseudo sextett, $1 \mathrm{H}, J=12.5 \mathrm{~Hz}$ and $J=3.6 \mathrm{~Hz}$, H-13), 4.1 (t, $\left.J=14 \mathrm{~Hz}, \mathrm{H}-2^{\prime}\right), 4.2$ (m, $\left.1 \mathrm{H}, \mathrm{H}-15\right)$, $4.7(\mathrm{~d}, \mathrm{~d}, J=5.2 \mathrm{~Hz}, J=9.8 \mathrm{~Hz}, \mathrm{H}-9), 5.5-5.7(\mathrm{~m}$, $3 \mathrm{H}, \mathrm{H}-2, \mathrm{H}-5, \mathrm{H}-7), 6.15$ (d, d, $1 \mathrm{H}, J=11.3 \mathrm{~Hz}$, $J=15.3 \mathrm{~Hz}, \mathrm{H}-4), 7.2(\mathrm{~m}, 1 \mathrm{H}, \mathrm{H}-3) \mathrm{ppm}$.

$$
\begin{aligned}
& \mathrm{C}_{46} \mathrm{H}_{76} \mathrm{O}_{10} \mathrm{~S}_{2}(852.9) \\
& \text { Calcd C } 64.8 \text { H 9.0, } \\
& \text { Found C } 64.5 \text { H 8.7. }
\end{aligned}
$$

7: positive FAB-MS $m / z=907\left(\mathrm{MNa}^{+}, 100 \%\right)$, ${ }^{1} \mathrm{H}$ NMR (pyridine- $\left.\mathrm{d}_{5}\right) \delta=2.55(\mathrm{~m}, 3 \mathrm{H}, \mathrm{H}-6$ and $\left.\mathrm{H}-1^{\prime}\right), 2.75(\mathrm{~d}, \mathrm{~d}, 1 \mathrm{H}, J=12.8 \mathrm{~Hz}, J=3.5 \mathrm{~Hz}$, H-12), 3.35 (pseudo sextett, $J=12.5 \mathrm{~Hz}$ and $J=$ $3.5 \mathrm{~Hz}, 1 \mathrm{H}, \mathrm{H}-13), 4.25(\mathrm{~m}, 1 \mathrm{H}, \mathrm{H}-15), 4.7$ (m, $1 \mathrm{H}, \mathrm{H}-9), 5.5-5.7(\mathrm{~m}, 3 \mathrm{H}, \mathrm{H}-2, \mathrm{H}-5, \mathrm{H}-7), 6.15(\mathrm{~d}$, d, $1 \mathrm{H}, J=15.3 \mathrm{~Hz}, J=11 \mathrm{~Hz}), 7.2(\mathrm{~m}, 1 \mathrm{H}, \mathrm{H}-3)$ ppm.

$$
\begin{array}{lll}
\mathrm{C}_{46} \mathrm{H}_{76} \mathrm{O}_{12} \mathrm{~S}_{2}(885.3) & \\
\text { Calcd } & \mathrm{C} 62.4 & \mathrm{H} 8.7, \\
\text { Found } & \mathrm{C} 62.7 & \mathrm{H} 8.8 .
\end{array}
$$

We would like to thank the BMFT for financial support, Dr. H. Kluge for valuable NMR-discussions and $\mathrm{H}$. Schneider for technical assistance.
[1] Part 2: S. Grabley, P. Hammann, W. Raether, J. Wink, and A. Zeeck, J. Antibiotics submitted (1989).

[2] M. Arcamone, C. Bertalozzi, M. Ghione, and T. Scotti, J. Microbiol. 7, 207 (1959).

[3] H. Kaiser and W. Keller-Schierlein, Helv. Chim. Acta 64, 407 (1981).

[4] K. Neupert-Laves and M. Dobler, Helv. Chim. Acta 65, 262 (1982).
[5] S. Takahashi, M. Arai, and E. Ohki, Chem. Pharm. Bull. 15, 1651 (1967).

[6] S. Takahashi, M. Arai, and E. Ohki, Chem. Pharm. Bull. 15, 1657 (1967).

[7] S. Takahashi and E. Ohki, Chem. Pharm. Bull. 15, 1726 (1967).

[8] D. Seebach, H. F. Chow, R. F. W. Jackson, M. A. Sutter, S. Thaisrivongs, and J. Zimmermann, Liebigs Ann. Chem. 1986, 1281. 